\title{
Determination of Surface and Thickness Characteristics of Textured Geomembranes Using Image Analysis
}

Nazli Yesiller

Associate Professor, Department of Civil and Environmental Engineering, Wayne State University, Detroit, MI 48202

Arif Cekic

Graduate Assistant, Department of Civil and Environmental Engineering, Wayne State University, Detroit, MI 48202.

ABSTRACT: Image analysis was used to determine surface topography and thickness of textured geomembranes. Images of cross sections (specimen length $\mathrm{x}$ thickness) of geomembranes were obtained at 50x magnification using a digital optical microscope.

Thickness was determined as the distance between the top and bottom surfaces along an entire cross section. For surface analysis, profiles of top and bottom surfaces were extracted from the cross sectionimages. Amplitude, spatial, hybrid, and functional texture parameters were determined. Tests were conducted on eleven samples of HDPE and LLDPE geomembranes manufactured by co-extrusion and embossing at varying thicknesses. The geomembranes were classified into three texture categories: high, medium, and low. Anisotropy and directionality were observed for all samples to varying degrees between and within manufacturing directions, respectively. Statistical analysis of the results indicated that surface topographyof geomembranes could be determined by analyzing one surface of five specimens with $50 \mathrm{~mm}$ length. Comparisons were made betweenimage analysis and mechanical tests for core thickness and asperity height. Image analysis and mechanical measurements were significantly different. Recommendations were made for the redesign of the mechanical devices bysizing the components in relation to the size and spacing of geomembrane texture features.

\section{Introduction}

Geomembrane surfaces are textured to provide improved interface performance against other geosynthetics or soils in various applications. Interface shear strength is a fundamental property in designing with geomembranes. Mechanical stability of various systems containing geomembranes depends on interface shear strength. Interface strength and shear resistance of geomembrane/geosynthetic and geomembrane/soil systems are significantly affected by surface texturecharacteristics of geomembranes. Thickness is a basic property of geomembranes that is used for general identification and classification of these materials. Mechanical and hydraulic performance of geomembranes are affectedbythickness. Thickness is used for manufacturing quality control as well as for conformance and acceptance in the field. 
This study was conducted to evaluate surface characteristics and thickness of textured geomembranes using image analysis and stan-dard mechanical test procedures. Variation of surface texture and thickness characteristics for specific geomembranes were deter-

mined, and comparisons of texture andthickness weremade between geomembranes manufactured using varying texturing processes. In addition, comparisons weremade between surface and thickness characteristics determined using image analysis and mechanical procedures.

\section{Background}

Surface texture characteristics of materials can be analyzed us-ing 2-D or 3-D analysis. Determination of 2-D parameters requires measurement of a profile along a surface of interest, whereas 3-D parametersrequiremeasurement of an entire surface. Some 3-D surface parameters may be estimated using 2-D profile measurements. Analyses may be conducted on a measured profile directly or at roughness, waviness, or form scales extracted from the mea-sured (raw) profile using filtering methods (Fig. 1). Existing quan-titative data for characterization of surface topographyof textured geomembranes wereobtained using 2-D profile measurements on HDPE samples. An optical technique was used to obtain profiles of textured geomembranes by Dove and Frost (1996), Dove et al. (1997), and Lee et al. (1998). Digitalimages of geomembrane cross sections wereobtained using an opticalmicroscope equipped with a CCD camera.Image analysis techniques wereused to obtain profiles along top and bottom surfaces of the geomembranes from the cross section images. A stylus profilometer was used to directly obtain profiles of textured geomembranes by Dove and Harpring (1999) and Dove et al. (2001).

Dove and Frost (1996)determined profile roughness parameter $(R L)$ and surface roughness parameter $(R S)$ for geomembranes. $R L$ is the quotient of the actual length of a profile to the projected length of a profile. $R S$ is the quotient of the actual area of a surface to the projected area of a surface. $R S$ was determined from measured 2-D profiles using the method described by Gokhale and Underwood (1990) and Gokhale and Drury (1990). Tests were conducted on two to five specimens with lengths of approximately $42 \mathrm{~mm}$ obtained from four textured geomembranes. The samples weredetermined to be oriented partially (not all surface features lie in a plane) and nonrandom (not all surface element orientations have an equal probability of occurrence). A textural classification system based on $R S$ was proposed that included four categories (smooth to heavily textured). It was determined that cross sections with a minimum length of $35 \mathrm{~mm}$ wererequired to obtain a steady measurement of $R S$ (Dove and Frost 1996). $R S$ was determined on several additional specimens with lengths of $50 \mathrm{~mm}$ obtained from the same four textured geomembranes (Dove et al. 1997; Lee et al. 1998). Overall, it is 
believed that on the order of twenty specimens were tested for each geomembrane type. However, systematic analyses of variation of $R S$ (or $R L$ ) for a single specimen or for specimens of the same type of geomembrane were not conducted. While Dove and Frost (1996) indicated that the variation of $R S$ was low, the reported values fromthis and the later studies indicated variations over $20 \%$.

Dove and Harpring (1999) and Dove et al. (2001) provided pa-rameters including amplitude (average roughness, maximum peak-to-valley height), spatial (average peak spacing), and hybrid (aver-age slope, RMS slope, average wavelength) parameters. Dove and Harpring (1999) used a total of four profiles each with a length of $40 \mathrm{~mm}$ to determine textureparameters for a geomembrane spec-imen in the machine direction. An extensive analysis of variation of textureparameters for a specific geomembrane specimen or a specific type of geomembrane was not included in the study. Dove et al. (2001) determined the manufacturing variability of surface characteristics of a textured geomembrane. Specimens $(300 \mathrm{~mm} \times 300 \mathrm{~mm}$ ) were obtained from a manufacturing facility every week over an approximately 8 -month period.Textureparameters were determined using four 40-mm-profile lengths on each specimen collected from the production line. The variation in the texture pa-rameters during the study period was between 14 and $29 \%$ with no discernable increasing or decreasingtrends with time. The variation of the texture parameters at a given sample collection time was not determined. The manufacturing variability of the texturing process for a particular time was not distinguished from the time-dependent variability of the texturing process.

Triplett and Fox (2001) provided qualitative assessment of sur-face characteristics of textured geomembranes in their study of shear strength of geomembrane/geosynthetic clay liner interfaces. Asperities were observed to be removed due to the shearing process from the geomembranes manufacturedusinglamination, whereas, the co-extruded geomembranes were intact subsequent to shearing. Also, variations were observed in the peak shear strength of the interfaces due to in-plane differences in the surface characteristics of the geomembranes along the same manufacturing direction ("forward" and "backward" test directions).

Thickness characteristics of textured geomembranes are deter-mined using two mechanical tests: corethickness and asperity height. Core thickness measurement is used to determine average minimumthickness for a textured geomembrane (ASTM D 5994, Test Method for Measuring Core Thickness of Textured Geomembrane). A specimen is placed horizontally in athickness gage between two measurement points (tips). The rounded conical tips are constructed with an apex of $60 \circ \pm 2 \circ$ and a radius of $0.8 \mathrm{~mm} \pm 0.1$ $\mathrm{mm}$ at the tip. The specimen is placed such that the locations of the tips coincide with the lowest points (valleys) on the surfaces of the specimen toresult in a low (core) thickness mea-surement.A constant average load of $0.56 \mathrm{~N}$ is used. Even though asperity height is 
generally classified under thickness determina-tion (Koerner 1998), it is used to obtain the maximum variation in elevation between the highest points (peaks) and the lowest points (valleys) on one surface of a textured geomembrane. Determination of asperity height is described in GRI Test Method GM12. In this method, a setting block with dimensions of $50 \mathrm{~mm}$ (length) $\times 20 \mathrm{~mm}$ (width) $\times 15 \mathrm{~mm}$ (height) is placed on a textured geomembrane. The setting block has a hole at the center of it, and a mea-surement probe with a diameter of $1.3 \mathrm{~mm}$ that is tapered to a contact "point" protrudes through this central hole in the block. The block is placed on a specimen such that it rests on the high points (peaks) on the specimen and the contact point rests on the lowest point (valley) on the surface of the specimen, providing a measurement of the localized "maximum" elevation differenceon the geomembrane. While specimens that are $75 \mathrm{~mm}$ in diameter are used for corethickness measurements, asperity height may be determined by placing the measurement device directly on a geomembrane roll without removing specimens. A total number of 10 specimens is used for corethickness measurements of a sample, and 10 measurements are made on a sample for asperity height de-termination. Manual adjustments are made in test locations to cap- ture peaks and valleys for both measurement types based on visual observation of a test material. Currently, standardized procedures are not available for determining surface characteristics of textured geomembranes.

Surface characteristics of textured geomembranes have been evaluated in a limited number of studies without comprehensive variability analyses. Published data are not commonly available forthickness and asperity height of textured geomembranes. Some of the geomembranes that were tested in previous studies are no longer manufactured, and data related to these are no longer relevant. This studywas conducted to provide an extensive analysis of surface andthickness characteristics of textured geomembranes. Initially, in-depth descriptions of textureparameters that can be used for geomembranes are provided. For experimental analyses, image analysis and standard mechanical test procedures wereused in the study.Variability of surface textureandthickness characteristics for specific geomembranes and variability between geomembranes producedbydifferent manufacturers were determined. Comparisons were made between image analysis and standard mechanical measurements. Recommendations for determination of surfaceand thickness characteristics are provided based on the results of the study.

\section{Texture Parameters}

The purpose ofthis section is to provide a concise description of surface topographyanalysis that may be used for textured geomembranes. The texture analysis is adapted from the surface metrology discipline. Texture parameters that are commonly used in surface metrology analysis include amplitude, spatial, hybrid, and functional 
parameters (Whitehouse 2002, 2003). These parameters may be determined on raw (unfiltered) profiles(referred to as preliminary profiles) directlyor at roughness, waviness, or form scales on filtered profiles (Fig. 1). Use of notation $R_{i}$ describes parameter " $i$ " determined at the roughness scale. $R_{i}$ has been used in various publications to describe texture parameters, since roughness scale is used commonly in surface metrology (Whitehouse 2002, 2003). This notation will be used inthis section to define texture parameters. The preliminary (unfiltered) parameters are shown using the notation $P_{i}$ and the waviness parameters are shown using the notation $W_{i}$. Existing quantitative data on textured geomembranes have been determined using preliminary profiles; however, notation $R_{i}$ has been used to describe the measured parameters in some cases. This causes confusion regarding the scale of analysis and should be avoided.

Equations for 2-D parameters are provided in this section for ease ofrepresentation. The common amplitude parameters include average roughness $\left(R_{a}\right)$; RMS roughness $\left(R_{q}\right)$; second and third mo-ments of height distribution, skewness $\left(R_{s k}\right)$ and kurtosis $\left(R_{k u}\right)$,respectively; maximum peak-to-valley height $\left(R_{t}\right)$; and average peak-tovalley height $\left(R_{z}\right)$. Average roughness is determined as:

$$
R_{a}=\frac{1}{L_{x}} \int_{0}^{L_{x}}|Z(x)| d x
$$

where $L_{x}$ is the length of a profile along a horizontal direction, $x$, and $Z(x)$ is the function representing the vertical height of the profile with respect to a horizontal best-fit line (with a mean height of zero). $R a$ is a common surfaceparameterr that is included in most texture肥 specifications used in the manufacturing industry. $R a$ quantifies the absolute magnitude of profile heights. $R q$ is the root mean square amplitude parameter. It has been shown to be better correlated to engineering function than $R a$ for variouss surfacess and

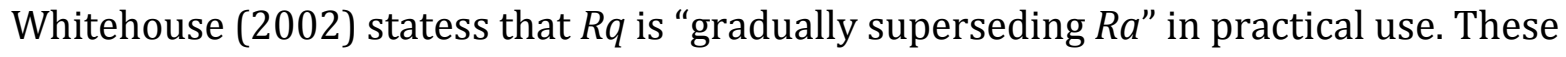
parameters are not sensitive to the distribution, magnitude, orr directionalal variations of peaks and valleys in a profile. Thee polarity of a profile is maintained in the skewness analysis and, therefore, Rsk may be used to assess the similarity of a surface (around a mean line). Rku may be used to determine the deviation of amplitude distribution for a surface from a normal distribution (Gaussian or bell curve distribution), which may be used to detect anomalies on a surface (Cohen 2002). A surface with normally distributed textures is expected to have an $R k u$ of 3 . The parameter may also be used to estimate the sharpness/bluntness of peaks or valleys in a profile (Whitehouse 2003). Softer features result in $R k u$ less than 3 and sharper features result in $R k u$ that are higher than 3. 
$R t$ is the maximum peak-to-valley height (differencece between the height of the highest peak and depth of the deepest valley), which needs to be determined on a high number of profiles to obtain statistically significant results. $R t$ is a divergent parameter (i.e., it increases as a greater profile length is investigated). $R t$ is the parameter intended to be measured using the mechanical asperity height measurement. An averaged peak-tovalley height $(R z)$ is used to provide a stable peak-to-valley amplitudee parameter for a given profile. This parameter is calculated as the average of the five highest measured peak-to-valley distances on the profile. Overall, the amplitude parameterss provide little information about spatial distribution of texture features on a surface. Nevertheles, theyy are commonly used in the manufacturing industryass quality control measures..

The two additional less common amplitude parameters are the profile roughness parameter $(R L)$ and the surface roughness parameter $(R S)$. Thee 3-D $R S s$ parameter can be determined from 2- D profiles using measurements on three cross sections oriented at 120 ॰ to each other with an error less than 6\%(Gokhale and Underwood 1990; Gokhale and Drury 1990). Both $R L$ and $R S S$ are general index parameters that provide overall measures of the magnitude of roughness of a surface. $R L$ is not as effective as $R a$ for characterizing the amplitude of a profile since $R L$ does not provide an average magnitude for profile heights. A similar comparison applies for 3-D analysis. $R L L$ and $R S s$ do not allow for determinationn of polarity of surfaces, spatial distribution of surface features, or variation of these featuress within or between manufacturing directions. It is believed that these parameters were used in the earlier studies of textured geomembranes (Dove and Frost 1996; Dove et al. 1997; Lee et al. 1998), sincee they are relatively easily determined index parameters. These parameters are not commonly used in surface metrology studies.

The spatial texture parameters are related to the spacing of sur-facefeatures. RSS is the average distance between local peaks and $R S m$ is the mean width of texture features.RSmrepresentss the aver-age distance between the positive/upward crossingss of the profile over the meanline. $R S S$ and $R S m$ are equivalent for simple periodic profiles. Countss of local peaks or valleys (with respect to the mean line of a profile or a specific percentage of the meanline) mayy also beprovided. Forr example, $R P c c$ is the number of peaks above the mean line for a given profile. Thee spatial parameters do not pro-vide information related to the amplitude characteristics of a profile (Whitehouse 2002).

The hybrid parameterss combine amplitude and spatialanalysis.. These parameters include average slope $(R A a)$; RMSS slope $(R A q)$; and average wavelength $(R \lambda q)$. The average slope is calculated as: 


$$
R_{\Delta a}=\frac{1}{L_{x}} \int_{0}^{L_{x}}\left|\frac{d(z)}{d(x)}\right| d x
$$

The RMS slope $(\mathrm{R} \Delta q)$ is the standard deciation of the average slope and is calculated as:

$$
R_{\Delta q}=\left[\frac{1}{L_{x}} \int_{0}^{L_{x}}\left(\frac{d Z(x)}{d x}-\left(\frac{d Z(x)}{d x}\right)\right)^{2} d x\right]^{1 / 2}
$$

where the term in the brackets, $<>$, represents the average value of all slopes along the profile direction. Analysis of distribution of slopes can be used to differentiate between peaks and valleys along varying directions (e.g., machine and cross-machine directions). Analysis of orientation of slopes can be used to distinguish forward and backward (i.e., $180^{\circ}$ apart-left to right and right to left along a profile) texture characteristics for a specific manufacturing direction. The variation of texturing in a given manufacturing direction can also be determined by investigating the differences in the cumulative length of the positive or negative slopes with respect to the projected horizontal length of the profile.

The averagewavelength $(R \lambda q)$ provides ameasure of the dominant spatial wavelength for a profile weighted by the amplitude of the components at varying wavelengths that comprise the profile. $R \lambda q$ can be used to differentiate between dominant long wavelength and short wavelength profile structures. $R \lambda q$ is calculated as:

$$
R_{\lambda q}=2 \pi \frac{R_{q}}{R_{\Delta q}}
$$

The functional parameters have generally been developed in relation to an intended use of a material. A set of functional parameters that can be used for textured geomembranes is material ratio (also referred to as bearing ratio) parameters. A material ratio curve is generated by assuming that a horizontal line is moving through a profile from the highest peak to the lowest valley (Fig. 2). The percentage of contact the horizontal line would make with the profile is quantified at each level. The curve provides the material-toair (or other material) ratio at any given depth. The y-axis is the total height of the profile (from the lowest valley to the highest peak), and the x-axis is the percent material above a corresponding height in a material ratio graph (Fig. $2 b$ ). $R k$ is the core roughness depth. The cutoff values used to determine $R k$ are based on the intersection points of the straight 
line that pass through the lowest slope portion of the material ratio curve and the vertical boundary lines corresponding to 0 and $100 \%$ on the x-axis. Mr1 and Mr2 closely approximate the upper and lower boundaries of the core roughness, respectively. Rpk represents the average height of the peaks above the core roughness. Rvk represents the average depth of the valleys below the core roughness. Material ratio parameters can be used for progressive determination of contact area (and thus contact stresses) with depth. The amount of material removed from the profile under applied load may be estimated, as well as interlocking of adjacent materials in the profile may be determined, using material ratio analysis. Special filtering methods that are originally developed for certain manufactured parts are used to obtain material ratio parameters (Whitehouse 2002, 2003). Profile section height $(P d c)$ is the core profile depth between fixed cutoff values of 20 and $80 \%$ determined on a material ratio curve developed using a preliminary profile.

\section{Testing Program}

The testing program consisted of determination of surface and thickness characteristics of textured geomembranes using image analysis and standard mechanical methods. Various surface and thickness characteristics were obtained and variations in these parameters were determined using statistical analysis. Comparisons were made between image analysis and standard mechanical measurements.

\section{Materials}

Tests were conducted on eleven geomembrane samples that represent two polymer types, HDPE and LLDPE, with nominal thicknesses ranging from 1 to $2.5 \mathrm{~mm}$ (Table 1 ). The geomembranes were manufactured using two primary texturing methods: co-extrusion and embossing. The materials labeled HG, HM, HP, LG, and LP are currently available (Fig. 3). The manufacturing methods for textured geomembranes have matured, and these products tested in the study have widespread use. The materials labeled HS were manufactured by a now defunct company and these (Fig. 3) are not currently available. These geomembranes were used in the test program, since they have been used in various applications and also since these materials have distinct texturing features in comparison with the other materials. This provided additional data for determining the effectiveness of the various texture parameters in differentiating between various types of textured geomembranes.

Tests were conducted on specimens with a length of $75 \mathrm{~mm}$ and width of $25 \mathrm{~mm}$. The 75-mm length was selected, since this is the size recommended for diameter of specimens for mechanical core thickness measurements. Rectangular specimens were used in the test program to allow for the use of same specimens in both image analysis and mechanical tests without any modifications to the specimens. This allows for direct comparison of the results obtained using the two test methods. A total of 20 specimens (10 
in the machine direction and 10 in the cross machine direction) were obtained from each geomembrane sample for testing the currently available materials. A total of 10 specimens ( 5 in the machine direction and 5 in the cross machine direction) were obtained from each geomembrane sample for testing the materials labeled HS, which are not currently available. Additional specimens were used to determine Rs for samples HG12, HM12, HP11, HS12, and LG11. These specimens were oriented 0, 120, and 240。 to the machine direction. In these tests, 3 specimens were obtained for each direction for each sample. Overall, a total of 235 specimens was used in the test program.

\section{Mechanical Thickness Tests}

Thickness characteristics of the specimens were initially determined using the core thickness and asperity height mechanical tests. Thickness and surface texture of the same specimens were then determined using image analysis. The core thickness was determined in accordance with ASTM D 5994, and the asperity height was determined in accordance with GRI GM-12. The specimens were traced extensively in attempts to obtain the "lowest" core thickness and the "highest" asperity height for a specimen. A total of 5 core thickness and 2 asperity height measurements were made on each specimen. Overall, 100 core thickness and 40 asperity height measurements were obtained for each of the HG, HM, HP, LG, and LP samples and 50 core thickness and 20 asperity height measurements were obtained for each of the HS samples.

\section{Image Analysis}

Image analysis tests were conducted subsequent to core thickness and asperity height determinations. Specimens were inserted vertically into specimen holders. The specimen holders consisted of a base plate and a circular ring attached to the base. Specimen holders with diameters of $150 \mathrm{~mm}$ were used to contain 2 or 3 specimens simultaneously (Fig. 4). Specimens were secured in place in narrow grooves that were machined into the base plate of the specimen holders. This configuration was used to ensure verticality of the specimens. The holders were then filled with Plaster of Paris mixtures prepared at a ratio of 2.5 parts Plaster of Paris: 1 part water to surround the specimens. The surrounding medium both aided in keeping the sample vertical as well as providing color contrast for image analysis. Upon hardening of the Plaster of Paris, the disk of hardened material was removed from the sample holder. The top surface of the disk was ground and polished using initially an electric sander and then by hand sanding to prepare the specimens for microscopy analysis. A 120-grit-sand paper was used to provide a smooth and level surface for the subsequent image analysis step. This specimen 
preparation approach is similar to the method used by Dove and Frost (1996). In this arrangement, a specimen is available for obtaining an image with the cross section of the material (thickness by specimen length) surrounded by the support/contrast medium.

The equipment used for image analysis consisted of an optical microscope equipped with a digital camera. The microscope had a magnification range of $25 \times$ to $300 \times$. Images with a resolution of $1280 \times 1024$ pixels were obtained with the camera. Preliminary images were obtained using magnifications of $25 \times$ to $100 \times$. Images of the specimens could not be obtained at magnifications higher than or equal to $100 \times$, as the field of view did not include the entire thickness of the specimen. A magnification of $50 \times$ was selected as this magnification provided both a high resolution $(8 \mu \mathrm{m}$ and $9 \mu \mathrm{m}$ along the length and thickness axes, respectively), and also allowed for a practical number of segments to be recorded along the length of a specimen. Approximately 9 segments were obtained for each specimen. These segments were then stitched to obtain the entire cross section of a specimen for further analysis. The entire recorded image of the cross section $(75-\mathrm{mm}$ length by thickness) was used to obtain the thickness for a specimen. Thickness was determined as the straight-line distance between the top and bottom sides of a specimen at a given measurement location. For each specimen, a total of 9600 thickness measurements were recorded across the entire length of the specimen. The cross-sectional images obtained were further processed to extract surface profiles along the top and bottom sides of the specimens (Fig. 5). Various texture parameters were then determined on the extracted profiles of the test specimens using a commercially available surface topography software.

\section{Results and Discussion}

Initially, surface analyses for the geomembranes are presented. Then, thicknesses of the geomembranes determined using the image analysis and mechanical methods are presented. Finally, comparisons of the measurements obtained using the two methods are provided. Comments about performance and general use of the methods are also included.

\section{Surface Texture Analysis}

Overall Trends - A total of 13 preliminary profile texture parameters is determined for the geomembranes (Table 2). Parameters determined along the machine and cross machine directions along the top surfaces of the specimens are provided for each geomembrane sample. For a geomembrane roll, top surface represents the surface that is 
"inside" a roll. The average (AVG), standard deviation (STD), and the coefficient of variation (COV) are provided for all of the parameters.

Positive skewnesses for all of the geomembranes indicated that the surface features tended to be more in the form of peaks than valleys. The kurtosis values were generally close to 3 indicating that the profile asperities (peaks and valleys) had near normal distributions. The average amplitude $(P a)$, maximum peak-to-valley height $(P t)$, slope $(P \Delta a)$, and section height $(P d c)$ of the raw profiles ranged between 0.13 and $0.22 \mathrm{~mm}, 0.61$ and $0.91 \mathrm{~mm}, 23.4$ and 35.3 degrees, and 0.25 and $0.39 \mathrm{~mm}$, respectively. The low values were generally associated with $\mathrm{HG}$ and LG samples, and the high values were generally associated with the HP and LP samples. The HM and HS samples generally had intermediate values. The RMS parameters $(P q$ and $P \Delta q)$ indicated similar trends as the average parameters $(P a$ and $P \Delta a)$. The number of peaks $(P P C)$ varied between $1.5 / \mathrm{mm}$ and $3.1 / \mathrm{mm}$. The low values were for HS samples. The surfaces of these materials consisted of alternating "bands" of textured and smooth regions. The low average values reflected the overall low amount of texturing on these samples compared to the other geomembranes. The high values were generally for HP samples. The average wavelength $(P \lambda q)$ varied between 1.38 and $3.46 \mathrm{~mm}$. The low values indicate short wavelengths and, thus, close spacing of texture features, and in contrast the high values indicate long wavelengths and distant spacing of texture features. The low values were measured for HP and LP samples, and the high values were measured for HS samples. The HM samples also had moderately high wavelengths. The texturing on these materials consists of more distinct and separated features compared to the more uniform texturing on HP, LP, HG, and LG samples. The peak spacing $(P S)$ and mean crossing $(P S m)$ also indicated similar trends. Textured geomembranes were classified into three categories: low texture, moderate texture, and high texture using amplitude, spatial, and hybrid parameters. The recommended ranges are provided in Table 3. The data indicate that the HP and LP samples have the highest overall texturing due to texture features that have high amplitude, low spacing, and high slope. The HG and LG samples have low texturing due to the low amplitude and slope. The HS samples

also have low texturing due to high spacing and low slope. The HM samples were determined to have moderate texturing due to moderate amplitude, spacing, and slope characteristics.

Analysis of the variation of the texture parameters indicated that high variations (multiple COVs $>20 \%$ ) were observed for high moments of amplitude distribution (skewness and kurtosis), spatial parameters, and hybrid parameters (wavelength). Low variations were observed for slope parameters (majority of COVs $<10 \%$ ). Loworder amplitude parameters generally had moderate variations. The HS samples generally had somewhat lower variability compared to the other samples, even though the number of specimens tested (five specimens) for each sample of these geomembraneswas lower than 
the number of specimens tested (ten specimens) for currently available geomembranes. This comparison was based on the count of COVs $<10 \%$ and COVs $>20 \%$ for each geomembrane sample (Table 2).

The texture parameters were also determined at the roughness and waviness scales (Cekic 2005). The profiles at these scales were obtained using filtering schemes that are commonly used in manufacturing industry. The material ratio parameters were also determined using common filtering options. These filtering options were available in the surface metrology software used in the test program. It was observed that the roughness profile had very small amplitudes, on the order of few tens of $\mu \mathrm{m}$. The material ratio parameters also had very small amplitudes that were not representative of the overall texturing of the geomembranes. The parameters determined at the waviness scale were somewhat similar to the parameters determined using the preliminary profile. The trends observed at these two scales were not significantly different from the trends obtained using the preliminary profile analysis. The distinct spacing of the texture features on the HS samples were more pronounced for the waviness scale analysis compared to the preliminary profile analysis. The waviness spacing and wavelength parameters were very high for these samples compared to the other samples.

Further analysis was conducted to determine the surface roughness parameter $(R S)$. The results were: 1.31 for HG12, 1.48 for HM12, 1.61 for HP11, 1.42 for HS12, and 1.28 for LG11. HG11 and LG11 were classified as "slightly textured," HM12 and HS12 were classified as "moderately textured," and finally HP11was classified as "heavily textured" according to the classification system proposed by Dove and Frost (1996). Similar classifications were made for the HG, LG, HM, and HP geomembranes using the surface texture parameters provided in Table 2 and recommended ranges provided in Table 3. However, the Dove and Frost (1996) classification for the HS geomembrane did not agree with the classification of the sample in the current study. $R S$ parameter was not effective in capturing the low texture of the HS geomembrane that results from the unique texture characteristics of this sample. Surface roughness provides only an overall indication of the amplitude of the texture features on a geomembrane with no indication of spacing, slope, or wavelength characteristics of surface features. Since the data obtained from the $R S$ analysis are rather simplistic and not fully effective in capturing texturing on a specimen, the authors believe that determination of this parameter is not warranted for texture analysis of geomembranes.

The predictive significance of the various parameters and measurement scales can be determined by establishing correlations to performance parameters, such as interface shear behavior. While preliminary profile analysis provides representative assessment of surface texture characteristics of geomembranes, the common filtering schemes that are 
used in the manufacturing industry may not be appropriate for textured geomembranes. Studies need to be conducted to develop filtering methods and additional analysis scales that are applicable to textured geomembranes as well as functional parameters that are related to the performance of these materials.

Isotropy and Directionality-Isotropy of textured surfaces is quantified by determining the relative proportions of representative parameters, $P a, P P c, P \_a$, and $P \lambda q$, in machine and cross machine directions (Table 4). Anisotropy was observed in the geomembranes based on all the measured parameters. The degree of anisotropy for the amplitude parameter $(P a)$ was higher than the spatial $(P P c)$ and hybrid parameters $(P \Delta a$ and $P \lambda q)$. The amplitude $(P a)$ and wavelength $(P \lambda q)$ parameters were generally lower in the machine direction than in the cross machine direction. The slope $(P \Delta a)$ and spatial $(P P C)$ parameters were generally higher in the machine direction than in the cross machine direction. Anisotropy of HM and HS samples was somewhat higher than the anisotropy of HG, LG, HP, and LP samples.

Directional characteristics of the geomembranes were further investigated by determining the cumulative length of the positive slopes (sections where the profile is inclined to the "right") and the cumulative length of the negative slopes (sections where the profile is inclined to the "left") for each manufacturing direction. These analyses were conducted from the "left" to the "right" side of the profiles obtained for all of the specimens. The directionality ratio $(D R)$ was calculated as the quotient of positive slopes to the negative slopes for each profile (Table 5). Directionality was observed for all of the geomembrane samples to varying degrees ( $D R$ between 0.7 and 1.6). $D R$ and variation between positive and negative slopes were lower for the machine direction than the cross machine direction for all of the samples except for the HP and LP samples, which demonstrated the opposite trend. The directionality as well as isotropy of geomembrane textures may be used in selection of the appropriate placement direction of geomembranes in the field for optimal interface shear strength.

Test Conditions - The test data were further investigated to determine the required test conditions for surface texture analysis of geomembranes. All of the texture parameters were determined for the bottom surfaces of the geomembranes subsequent to the determination of the parameters for the top surfaces (presented above). The variation of the parameters was determined between the top and bottom surfaces. The variation between the two surfaces was less than $10 \%$ for $77 \%$ of the texture data, and the variation was less than $15 \%$ for $90 \%$ of the texture data. It was observed that the highest variations (up to $27 \%$ ) were obtained for the HS geomembranes. The top/bottom variation analysis 
was repeated for the texture data, excluding these samples. For the currently available geomembranes, the variation between the two surfaces was less than $10 \%$ for $82 \%$ of the data, and the variation was less than $15 \%$ for $95 \%$ of the texture data, with no variations over $19 \%$. Overall, $85 \%$ of the top and bottom surface data were statistically similar based on t-tests conducted at $95 \%$ significance level.

The amplitude parameters $P a$ and $P q$ and the slope parameters $P \Delta a$, and $P \Delta q$ were higher for the top surfaces than for the bottom surfaces of all of the geomembranes. There were no significant, consistently lower or higher trends for the other texture parameters. In addition, the trends observed in the numerical values and variations of the texture parameters for the bottom surface of the geomembranes were similar to the trends observed for the top surface of the geomembranes. In fact, the variability of the texture parameters was somewhat lower for the bottom surface than the top surface. The textural classification of the geomembranes based on the bottom surface measurements was the same as the classification based on top surface measurements. Further analysis was conducted by averaging the top and bottom surface measurements. The trends in the averaged data were similar to the trends in the individual surface data. Therefore, it was concluded that surface texture analysis could be conducted using only top or bottom surface measurements or using the average of the top and bottom surface measurements for geomembranes. Detailed statistical parameters for the bottom surface texture analysis and averaged top and bottom surface texture analysis are provided in Cekic (2005). In cases with significant differences in manufacturing conditions (e.g., die settings, extruder settings, type of resin, etc.) between the top and bottom surfaces of a geomembrane, both surfaces may need to be tested for surface characteristics.

The size and number of specimens required for surface texture analysis were also investigated. The texture parameters were determined using randomly selected sections along the full length of the 75-mm-long specimens. The sections evaluated had lengths of $10 \mathrm{~mm}, 20 \mathrm{~mm}, 30 \mathrm{~mm}, 40 \mathrm{~mm}$, and $50 \mathrm{~mm}$. The analysis was conducted using the data obtained for the top surface of the specimens of the eight currently available geomembranes. Selected amplitude, spacing, and hybrid texture parameters, $P a, P t, P S$, $P \Delta a, P \Delta q$, and $P \lambda q$, determined using the shorter lengths, were compared with the parameters determined on the full-length specimens. The comparisons were made using ttests. The results indicated that $0,5,26$, and $54 \%$ of the data obtained on $10-\mathrm{mm}, 20-\mathrm{mm}$, 30- $\mathrm{mm}$, and 40-mm sections, respectively, were similar to parameters determined on the 75-mm-long specimens. For $50-\mathrm{mm}$ sections, $91 \%$ of the texture data were statistically similar to parameters determined on the 75-mm-long specimens. Data from the currently available geomembranes were also used for analyzing the number of specimens required for surface texture analysis. Texture parameters were determined using five randomly selected specimens out of the ten total specimens obtained for a specific manufacturing 
direction. The analysis indicated that $99 \%$ of the texture parameters determined using five specimens were statistically similar to the parameters determined using ten specimens for a given geomembrane sample. Detailed statistical parameters for the size and number of specimen analyses are provided in Cekic (2005). All of the comparisons were made using ttests at $95 \%$ significance level. It was concluded that representative surface texture analysis for geomembranes can be conducted using five specimens with 50 -mm length.

\section{Thickness Characteristics}

The thicknesses determined using image analysis are presented in Fig. 6. For each type of geomembrane, the thickness distributions obtained for all of the specimens of the particular type of geomembrane were averaged and are presented in the figure. Statistical parameters for the thickness data are provided in Table 6. The variations of the average thicknesses were low (COVs range between 1 and 4\%) for a particular geomembrane. The variability of average thickness of textured geomembranes is similar to the variability of average thickness of smooth geomembranes determined in previous study (Yesiller and Cekic 2001). Variation of the thickness data for each thickness interval presented in Fig. 6 was also determined for each geomembrane sample. The variation in the frequency of occurrence of the majority of the data in the central histogram columns was approximately $20 \%$. The variations for the low and high thickness ends were higher (details of this extensive analysis are provided in Cekic (2005)). While the variations of average thicknesses were low, the variations of the distributions of thicknesses were high for a particular geomembrane. These values represent the expected variability of thickness for textured geomembranes.

The thickness distributions for all of the geomembranes are similar with significant skew in the data towards the tail end (high thicknesses above the average thickness), as indicated by the positive skewness values. The kurtosis values that are higher than 3 for all samples also indicate the non-normal distribution (skewed distribution) of the thickness data. The majority ( $80 \%$ ) of the thickness data is within the range of nominal thickness to 25 to $50 \%$ higher than the nominal thickness. The range for the majority of data decreases as the thickness of geomembrane samples increases. This indicates that the manufacturing processes generate texturing that is independent of the thickness of the geomembranes. A relatively low amount of data ( 1 to $8 \%$ ) lies below the nominal thickness of the geomembranes. The lowest percentages for data below nominal thickness were obtained for embossed HM samples, whereas the highest percentages were obtained for the coextruded HP samples. Thickness histograms such as the examples presented in Fig. 6 can be used as quality control tools for textured geomembranes. Representative target 
thickness distributions can be developed for a given manufacturing process, and the compliance of the manufactured products to the target distributions can be determined.

The average (AVG), standard deviation (STD), and the coefficient of variation (COV) are provided for image analysis "core thickness" and mechanical core thickness measurements in Table 7. Results from all machine and cross machine direction specimens are provided for each type of geomembrane. For image analysis, the lowest thickness measured on each specimen of a particular sample of geomembrane is used in the analysis. This thickness represents the ultimate lowest value that is targeted to be determined using the mechanical core thickness measurements. An analysis was also conducted using the five lowest thicknesses determined on each specimen using image analysis. However, this analysis (the thickness values and associated statistics) was essentially the same as the analysis using the single lowest thickness value and, thus, is not presented herein.

Analyses of the test results indicate that the mechanical core thicknesses are 6 to $22 \%$ higher than the image analysis core thicknesses for the geomembranes (Table 7). The core thicknesses determined using mechanical analysis are higher than the nominal thicknesses of geomembrane samples, whereas the image analysis core thicknesses are lower than the nominal thicknesses of the geomembrane samples (Table 7). A t-test was conducted to determine the similarity of core thicknesses determined using the two methods for each geomembrane sample at 95\% significance level. Results of these tests indicated that mechanical and image analysis core thicknesses were statistically dissimilar. The reasons for the low image analysis and high mechanical core thickness measurements are twofold. The most important reason is the configuration (size and shape) of the mechanical probe tips. Even though the measurement tips are tapered to have small radii, they still do not fit into the valleys that are present on the surface of the textured geomembranes. The combination of the diameter of the probe and taper length in comparison to the average peak spacing $(P S)$ and the average peak-to-valley height $(P z)$, respectively, prevents the probe from reaching the lowest valleys. The probe used in this study had a diameter of $5 \mathrm{~mm}$, which was tapered to the rounded probe tip with a radius of $0.8 \mathrm{~mm}$ over a distance of approximately $3 \mathrm{~mm}$. The diameter of the probe at a distance of $0.59 \mathrm{~mm}$ from the probe tip (corresponding to average peak-to-valley height for all of the samples) is $1.61 \mathrm{~mm}$. This diameter is larger than the average localized peak spacing (PS) for all of the currently manufactured geomembranes (Table 2). For HS geomembranes, this diameter is smaller than the average $P S$; however, the surface features on these materials occur in bands and it is believed that the average spacings within the textured regions are smaller than $1.61 \mathrm{~mm}$. In summary, the probe tips could not physically fit into the valleys between the peaks on the surface of the geomembranes tested in the study. In addition, the location of the lowest thickness in a geomembrane is not readily available. While the ASTM standard recommends adjusting a specimen in a thickness measurement device until 
valleys are located to obtain a core thickness measurement, it is not possible to estimate visually the location with the lowest thickness. The roaming procedure does not guarantee finding the location with the lowest thickness.

The average (AVG), standard deviation (STD), and the coefficient of variation (COV) are provided for image analysis "asperity height" and mechanical asperity height measurements in Table 8. Similar to core thickness analysis, results from all machine and cross machine direction specimens are provided for each type of geomembrane. For image analysis, the maximum peakto- valley heights $(P t)$ measured on each specimen of a particular sample of geomembrane are provided. This height represent the ultimate asperity height value that is targeted to be determined using the mechanical measurements. Image analysis and mechanical measurements were both made on the top surface of the specimens.

Analyses of the test results indicate that the image analysis asperity heights are 17 to $42 \%$ higher than the mechanical asperity heights for the geomembranes (Table 8). A ttest was conducted to determine the similarity of asperity heights determined using the two methods for each geomembrane sample at 95\% significance level. Results of these tests indicated that mechanical and image analysis asperity heights were statistically dissimilar. The combination of the diameter of the probe and taper length in comparison to the average peak spacing $(P S)$ and the maximum peak-to-valley height $(P t)$, respectively, prevents the probe from reaching the lowest valleys. The probe has a diameter of $1.3 \mathrm{~mm}$ that is tapered to a contact "point" over an approximately 0.76-mm length (length not specified in the GRI GM12 standard). The 0.76-mm length is shorter than the majority of the maximum peak-to-valley heights $(P t)$ and the diameter of the probe $(1.3 \mathrm{~mm})$ is larger than all of the localized peak spacings for the currently available geomembranes. In summary, similar to core thickness device, the asperity height probe tip could not physically fit into the valleys between the peaks on the surface of the geomembranes tested in the study. In addition, the setting block rests on the highest peaks under its area along a plane that may or may not be in the horizontal plane. This arrangement results in the potential deviation of the measurement probe from the vertical, which also prevents the probe from fully reaching the low valleys. Similar to core thickness determination, the location of the setting block is adjusted during a measurement to locate the highest peaks and deepest valley. However, this procedure does not guarantee finding such locations.

Overall, it was observed that the mechanical measurements are not entirely effective in capturing the thickness and surface texture properties of textured geomembranes. The dimensional properties and configuration of the mechanical devices are not conducive to capturing the lowest thickness and highest peak-to-valley heights for geomembranes. The setups used in the mechanical tests have simplistic designs that have been developed without considering the surface texture characteristics of the 
geomembranes. In addition, the discrete nature of these measurements prevents capturing the desired limiting values for these materials. While the mechanical tests are easy to conduct, their effectiveness in determining the thickness and texture properties of geomembranes is limited. The core thickness and asperity height devices can be improved by adjusting the size and the shape of the measurement probes and tips based on the peak spacing and peak-to-valley height of the geomembranes. A diameter of less than $0.6 \mathrm{mmover}$ a probe length of more than $0.9 \mathrm{~mm}$ at the tip of a probe is recommended for mechanical devices. Another consideration is related to the overall need for determination of asperity height of textured geomembranes. Additional texture parameters may be better correlated to engineering properties and response of textured geomembranes than asperity height, and therefore, there may be need to develop methods to determine these parameters.

The image analysis method described in this paper provides representative measurements for thickness and surface texture properties of geomembranes; however, the method is not highly practical for routine use. It is believed that the image analysis method used in the study allows for capturing all of the texture features on a geomembrane. Nondestructive test methods that use laser, ultrasonic, magnetic, or profilometry techniques may present effective alternatives that provide continuous measurements for thickness and surface characteristics. These alternative methods may not be as effective as the imaging method in capturing intricate features (e.g., features with hidden air pockets) on geomembrane surfaces.

\section{Summary and Conclusions}

Surface texture and thickness characteristics of textured geomembranes were evaluated using image analysis and standard mechanical test procedures. Images of cross sections (length by thickness) of textured HDPE and LLDPE geomembranes were obtained using a digital optical microscope. Texture characteristics of the geomembranes were determined using a commercially available surface metrology software. Variation of surface texture and thickness characteristics for specific geomembranes was determined and comparisons were made for surface and thickness characteristics between geomembranes manufactured using varying texturing processes. Comparisons were also made between surface and thickness characteristics determined using mechanical and image analysis procedures.

Analyses of raw (unfiltered preliminary) profiles were used to determine surface texture properties of the geomembranes. The texture parameters for the various geomembranes tested were statistically dissimilar. Geomembranes were classified into three categories based on amplitude, spatial, and hybrid texture parameters: low, 
moderate, and high surface texture. Anisotropy and directionality were observed for all samples to varying degrees between manufacturing directions and within a particular manufacturing direction, respectively. These parameters can be used in the placement of geomembranes in the field for optimum interface shear resistance. Statistical analysis of the test results indicated that surface topography of textured geomembranes could be determined by analyzing one surface of five specimens with 50-mm length.

Image analysis allows for determination of texture and thickness characteristics of geomembranes along the entire surface and cross section of the geomembranes, respectively. Thickness histograms can be generated for a manufacturing process and can be used as quality control tools for textured geomembranes. The limiting thickness values (maxima or minima) and average thicknesses can be determined using image analysis. Mechanical test methods provide discrete measurements and limiting values are not effectively captured using these tests. The measured core thicknesses were higher and measured asperity heights were lower in mechanical tests than in image analysis tests. Mechanical measurements are simpler than the image analysis measurements; however, the representativeness of the data obtained in these tests is low due to the size and shape limitations of the devices. The configuration of the mechanical devices can be adjusted using surface characteristics of textured geomembranes. A diameter of less than $0.6 \mathrm{~mm}$ over a probe length of more than $0.9 \mathrm{~mm}$ at the tip of a probe is recommended for the devices.

\section{Acknowledgment}

Partial financial support for this study was provided by the National Science Foundation (NSF Grant No: CMS 97-01947). Materials were supplied by various past and present manufacturers, including GSE Inc., Agru-America Inc., Poly-Flex Inc., and National Seal Company.

\section{References}

ASTM Standard D 5994-98, 2001: Test Method for Measuring Core Thickness of Textured Geomembrane, Annual Book of ASTM Standards, ASTM International, West Conshohocken, PA.

GRI Standard GM12, 2001: Standard Test Method for Asperity Measurement of Textured Geomembranes Using a Depth Gage, GRI Test Methods. 
Cekic, A., to be completed in 2005, "Nondestructive Testing and Image Analysis of Geomembranes," Ph.D. Thesis, Department of Civil and Environmental Engineering,Wayne State University, Detroit, MI.

Cohen, D. K., 2002, "Glossary of Surface Texture Parameters," Michigan Metrology, LLC Application Notes.

Dove, J. E., Adams, M.W., and Johnson, M. L., 2001, "Manufacturing Variability of Coextruded Geomembrane Surface Texture," Proceedings, Geosynthetics Conference 2001, North American Geosynthetics Society, pp. 823-834.

Dove, J. E. and Frost, J. D., 1996, “A Method for Measuring Geomembrane Surface Roughness," Geosynthetics International, IFAI, Vol. 3, No. 3, pp. 369-392.

Dove, J. E., Frost, J. D., Han, J., and Bachus, R. C., 1997, “The Influence of Geomembrane Surface Roughness on Interface Strength," Proceedings of Geosynthetics '97, North American Geosynthetics Society, pp. 863-876.

Dove, J. E. and Harpring, J. C., 1999, "Geometric and Spatial Parameters for Analysis of Geomembrane/Soil Interface Behavior," Proceedings of Geosynthetics '99, North American Geosynthetics Society, pp. 575-588.

Gokhale, A. M. and Drury,W. J., 1990, "A General Method for Estimation of Fracture Surface Roughness: Part II. Practical Considerations," Metallurgical Transactions A, Vol. 21A, pp. 1201- 1207.

Gokhale, A. M. and Underwood, E. E., 1990, "A General Method for Estimation of Fracture Surface Roughness: Part I. Theoretical Aspects," Metallurgical Transactions A, Vol. 21A, pp. 1193- 1199.

Koerner, R. M., 1998, Designing with Geosynthetics, Prentice-Hall, Upper Saddle River, NJ.

Lee, S.W., Frost, J. D., and Righter, G. K., 1998, “The Influence of Geomembrane Surface Roughness on Geomembrane-Geotextile Interface Strength," Proceedings of the 6th International Conference on Geosynthetics, pp. 433-438.

Triplett, E. J. and Fox, P. J., 2001, "Shear Strength of HDPE Geomembrane/Geosynthetic Clay Liner Interfaces," Journal of Geotechnical and Geoenvironmental Engineering, ASCE, Vol. 127, No. 6, pp. 543-552.

Whitehouse, D. J., 2002, Surfaces and Their Measurement, Taylor and Francis, New York.

Whitehouse, D. J., 2003, Handbook of Surface and Nanometrology, IOP Publishing, London.

Yesiller, N. and Cekic, A., 2001, "Determination of Thickness of Smooth Geomembranes," Geotechnical Testing Journal, Vol. 24, No. 4, December, pp. 359-369. 


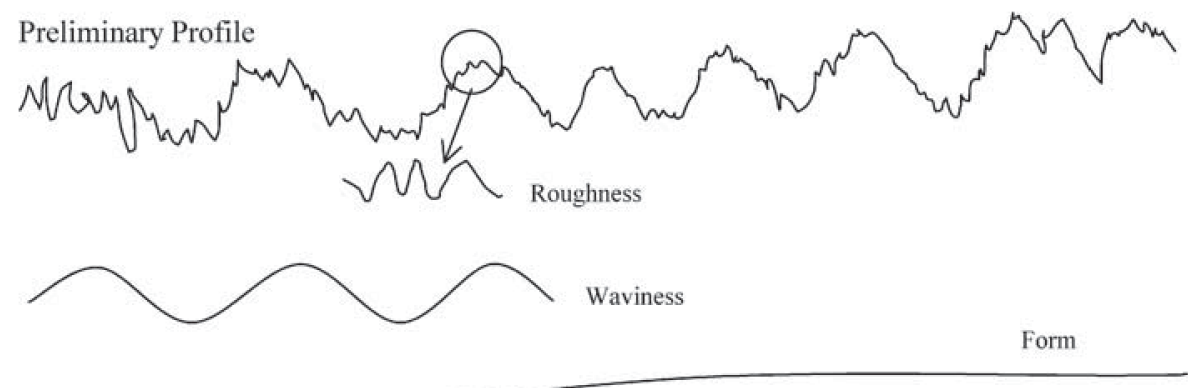

FIG. 1 -Scales of measurement (adapted from Whitehouse 2002).

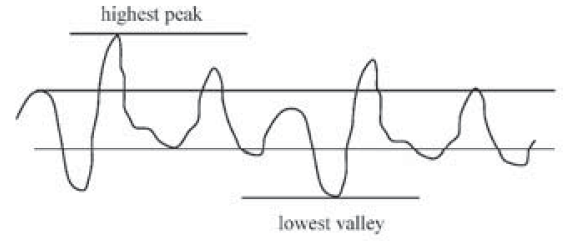

(a)

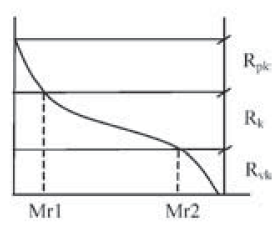

(b)

FIG. 2-Material ratio parameters.

TABLE 1-Materials used in the test program.

\begin{tabular}{|c|c|c|c|}
\hline Sample & Polymer & $\begin{array}{c}\text { Nominal } \\
\text { Thickness, } \\
\text { mm }\end{array}$ & $\begin{array}{c}\text { Manufacturing } \\
\text { Method }\end{array}$ \\
\hline HG11 & $\mathrm{HDPE}^{a}$ & 1.0 & Co-extrusion \\
\hline HG12 & HDPE & 1.5 & Co-extrusion \\
\hline HM11 & HDPE & 1.0 & Embossing \\
\hline HM12 & HDPE & 1.5 & Embossing \\
\hline HP11 & HDPE & 1.5 & Co-extrusion \\
\hline HP12 & HDPE & 2.0 & Co-extrusion \\
\hline HS11 & HDPE & 1.0 & Co-extrusion \\
\hline HS12 & HDPE & 1.5 & Co-extrusion \\
\hline HS13 & HDPE & 2.5 & Co-extrusion \\
\hline LG11 & $\mathrm{LLDPE}^{b}$ & 1.0 & Co-extrusion \\
\hline LP11 & LLDPE & 1.0 & Co-extrusion \\
\hline
\end{tabular}

${ }^{a}$ High Density Polyethylene.

${ }^{b}$ Linear Low Density Polyethylene. 


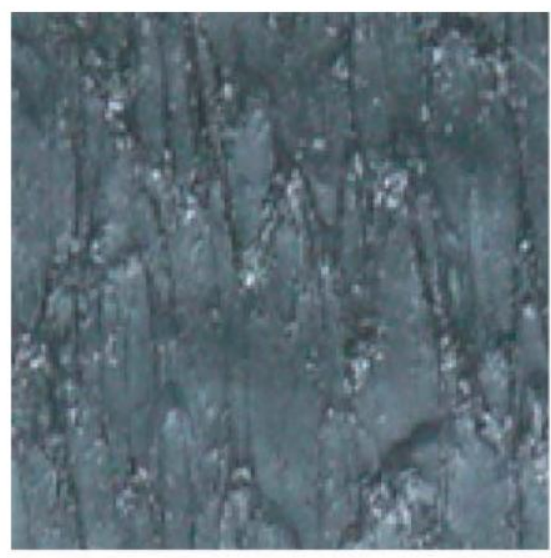

a) HG Geomembrane

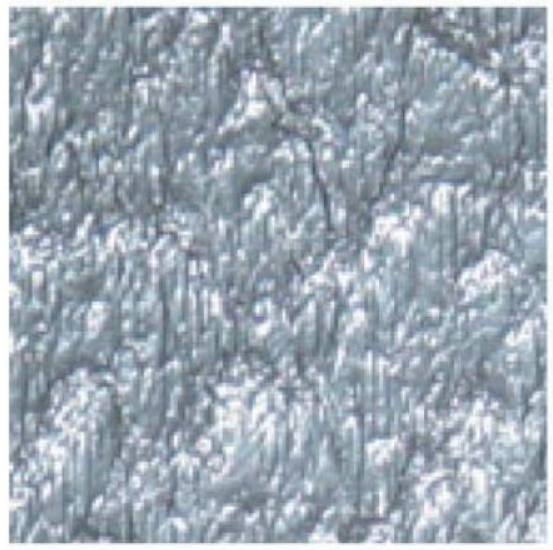

c) HP Geomembrane

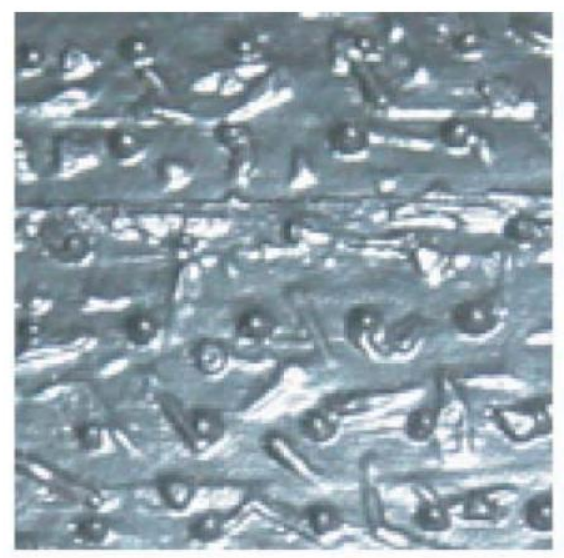

b) HM Geomembrane

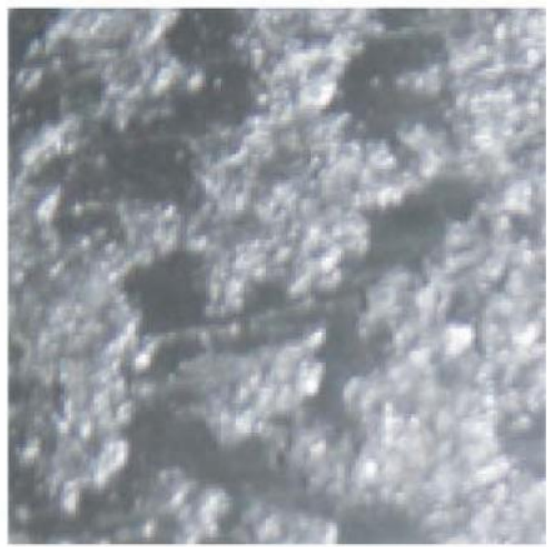

d) HS Geomembrane

FIG. 3-Geomembrane samples. 


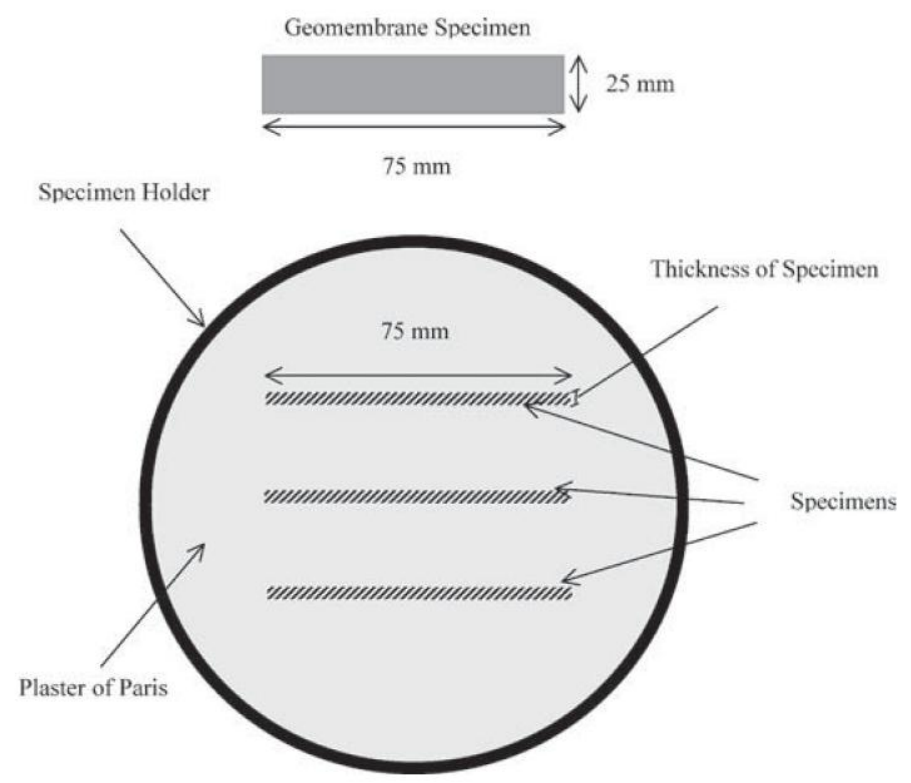

FIG. 4 Geomembrane specimen and specimen holder.

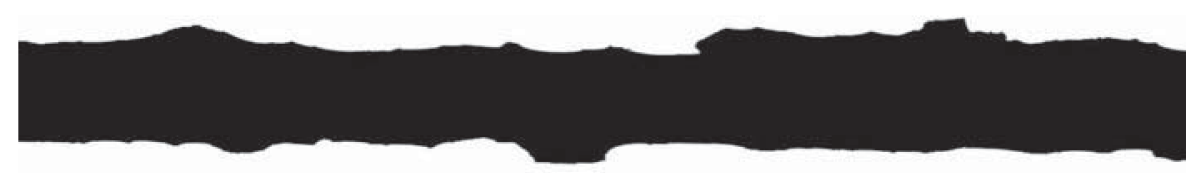

Cross Section Image Used for Determination of Thickness

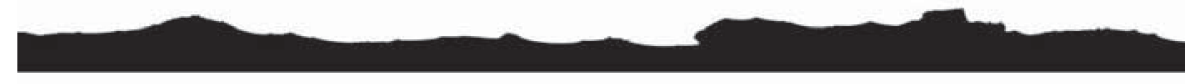

Isolated Top Half of Cross Section Image

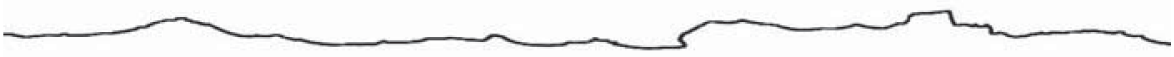

Extracted Profile Used for Texture Analysis

FIG. 5 - Image analysis of specimens. 
TABLE 2-Texture parameters.

\begin{tabular}{|c|c|c|c|c|c|c|c|c|c|c|c|c|c|c|}
\hline GM & Statistic & $P_{a}{ }^{a}$ & $P_{q}{ }^{a}$ & $P_{s k}^{a}$ & $P_{k u}{ }^{a}$ & $P_{t}^{a}$ & $P_{z}^{a}$ & $P S_{m}^{a}$ & $P S^{a}$ & $P_{\Delta a}^{b}$ & $P_{\Delta q}^{b}$ & $P P_{c}^{c}$ & $P_{\lambda q}{ }^{a}$ & $P_{d c^{a}}^{a}$ \\
\hline \multirow[t]{3}{*}{$\mathrm{HG}_{11^{d}}$} & AVG & 0.135 & 0.177 & 0.654 & 3.475 & 0.641 & 0.505 & 1.698 & 0.833 & 25.750 & 37.650 & 2.816 & 1.782 & 0.265 \\
\hline & STD & 0.019 & 0.016 & 0.154 & 0.431 & 0.083 & 0.069 & 0.572 & 0.144 & 2.927 & 2.901 & 0.384 & 0.262 & 0.039 \\
\hline & $\operatorname{COV}(\%)$ & 14.0 & 9.2 & 23.6 & 12.4 & 13.0 & 13.6 & 33.7 & 17.3 & 11.4 & 7.7 & 13.6 & 14.7 & 14.9 \\
\hline \multirow[t]{3}{*}{ HG11 ${ }^{e}$} & AVG & 0.128 & 0.164 & 0.571 & 2.904 & 0.613 & 0.511 & 1.795 & 0.854 & 24.790 & 36.830 & 2.423 & 1.822 & 0.245 \\
\hline & STD & 0.020 & 0.024 & 0.180 & 0.526 & 0.059 & 0.061 & 0.210 & 0.123 & 2.422 & 2.251 & 0.515 & 0.202 & 0.033 \\
\hline & $\operatorname{COV}(\%)$ & 15.4 & 14.4 & 31.5 & 18.1 & 9.6 & 11.9 & 11.7 & 14.4 & 9.8 & 6.1 & 21.2 & 11.1 & 13.6 \\
\hline \multirow[t]{3}{*}{ HG12 } & AVG & 0.125 & 0.165 & 0.811 & 3.608 & 0.827 & 0.602 & 1.735 & 0.910 & 25.090 & 37.320 & 2.470 & 1.952 & 0.257 \\
\hline & STD & 0.019 & 0.017 & 0.240 & 0.739 & 0.073 & 0.068 & 0.301 & 0.178 & 1.936 & 2.122 & 0.373 & 0.299 & 0.045 \\
\hline & $\operatorname{COV}(\%)$ & 15.2 & 10.5 & 29.5 & 20.5 & 8.9 & 11.3 & 17.3 & 19.6 & 7.7 & 5.7 & 15.1 & 15.3 & 17.5 \\
\hline \multirow[t]{3}{*}{ HG12 } & AVG & 0.125 & 0.166 & 0.735 & 3.645 & 0.726 & 0.545 & 1.794 & 0.988 & 27.690 & 39.670 & 2.547 & 2.015 & 0.250 \\
\hline & STD & 0.017 & 0.018 & 0.213 & 0.651 & 0.086 & 0.062 & 0.281 & 0.253 & 3.303 & 3.270 & 0.537 & 0.449 & 0.044 \\
\hline & $\operatorname{cov}(\%)$ & 13.6 & 11.0 & 29.0 & 17.9 & 11.8 & 11.4 & 15.6 & 25.6 & 11.9 & 8.2 & 21.1 & 22.3 & 17.5 \\
\hline \multirow[t]{3}{*}{ HM11 } & AVG & 0.135 & 0.183 & 0.694 & 3.490 & 0.790 & 0.576 & 2.503 & 1.563 & 28.980 & 40.320 & 2.289 & 2.813 & 0.272 \\
\hline & STD & 0.021 & 0.020 & 0.254 & 0.767 & 0.060 & 0.047 & 0.568 & 0.514 & 2.564 & 2.841 & 0.378 & 0.671 & 0.038 \\
\hline & $\operatorname{COV}(\%)$ & 15.2 & 11.1 & 36.6 & 22.0 & 7.6 & 8.2 & 22.7 & 32.9 & 8.8 & 7.0 & 16.5 & 23.9 & 13.9 \\
\hline \multirow[t]{3}{*}{ HM11 } & AVG & 0.156 & 0.199 & 0.877 & 3.588 & 0.791 & 0.580 & 2.232 & 1.363 & 26.320 & 37.310 & 2.121 & 2.738 & 0.319 \\
\hline & STD & 0.021 & 0.022 & 0.184 & 0.553 & 0.102 & 0.093 & 0.558 & 0.431 & 3.238 & 2.777 & 0.306 & 0.703 & 0.039 \\
\hline & $\operatorname{COV}(\%)$ & 13.3 & 11.1 & 21.0 & 15.4 & 12.9 & 16.0 & 25.0 & 31.7 & 12.3 & 7.4 & 14.4 & 25.7 & 12.4 \\
\hline \multirow[t]{3}{*}{ HM12 } & AVG & 0.149 & 0.200 & 0.737 & 3.454 & 0.758 & 0.584 & 1.865 & 1.117 & 29.800 & 41.310 & 2.147 & 2.065 & 0.302 \\
\hline & STD & 0.028 & 0.026 & 0.191 & 0.672 & 0.125 & 0.099 & 0.218 & 0.231 & 2.553 & 2.342 & 0.266 & 0.212 & 0.049 \\
\hline & $\operatorname{COV}(\%)$ & 18.6 & 13.1 & 25.9 & 19.5 & 16.5 & 17.0 & 11.7 & 20.7 & 8.6 & 5.7 & 12.4 & 10.3 & 16.3 \\
\hline \multirow[t]{3}{*}{ HM12 } & AVG & 0.173 & 0.222 & 0.863 & 3.740 & 0.818 & 0.624 & 2.049 & 1.315 & 29.440 & 41.490 & 2.153 & 2.313 & 0.344 \\
\hline & STD & 0.037 & 0.037 & 0.200 & 0.728 & 0.093 & 0.063 & 0.413 & 0.351 & 2.775 & 3.341 & 0.223 & 0.489 & 0.075 \\
\hline & $\operatorname{COV}(\%)$ & 21.4 & 16.9 & 23.2 & 19.5 & 11.3 & 10.0 & 20.2 & 26.7 & 9.4 & 8.1 & 10.4 & 21.2 & 21.8 \\
\hline HP11 & AVG & 0.170 & 0.230 & 0.627 & 3.310 & 0.832 & 0.619 & 1.321 & 0.743 & 33.710 & 46.020 & 3.128 & 1.616 & 0.336 \\
\hline & STD & 0.023 & 0.024 & 0.136 & 0.503 & 0.036 & 0.046 & 0.263 & 0.094 & 2.366 & 1.991 & 0.284 & 0.201 & 0.033 \\
\hline & $\operatorname{COV}(\%)$ & 13.4 & 10.5 & 21.6 & 15.2 & 4.4 & 7.4 & 19.9 & 12.7 & 7.0 & 4.3 & 9.1 & 12.5 & 9.8 \\
\hline HP11 & AVG & 0.216 & 0.275 & 0.748 & 3.521 & 0.883 & 0.647 & 1.201 & 0.622 & 33.590 & 45.740 & 3.050 & 1.375 & 0.387 \\
\hline & STD & 0.030 & 0.029 & 0.220 & 0.831 & 0.056 & 0.057 & 0.364 & 0.185 & 2.505 & 2.307 & 0.283 & 0.359 & 0.026 \\
\hline & $\operatorname{COV}(\%)$ & 14.0 & 10.5 & 29.4 & 23.6 & 6.3 & 8.8 & 30.3 & 29.7 & 7.5 & 5.0 & 9.3 & 26.1 & 6.7 \\
\hline HP12 & AVG & 0.200 & 0.258 & 0.852 & 3.876 & 0.896 & 0.677 & 1.393 & 0.807 & 34.730 & 47.130 & 3.046 & 1.576 & 0.378 \\
\hline & STD & 0.029 & 0.026 & 0.157 & 0.589 & 0.106 & 0.075 & 0.346 & 0.281 & 3.312 & 2.821 & 0.277 & 0.372 & 0.039 \\
\hline & $\operatorname{COV}(\%)$ & 14.4 & 10.0 & 18.4 & 15.2 & 11.8 & 11.1 & 24.8 & 34.8 & 9.5 & 6.0 & 9.1 & 23.6 & 10.3 \\
\hline HP12 & AVG & 0.197 & 0.254 & 0.930 & 3.856 & 0.906 & 0.640 & 1.243 & 0.651 & 33.340 & 45.160 & 2.907 & 1.444 & 0.378 \\
\hline & STD & 0.032 & 0.032 & 0.156 & 1.283 & 0.091 & 0.058 & 0.279 & 0.159 & 2.017 & 2.031 & 0.343 & 0.299 & 0.049 \\
\hline & $\operatorname{COV}(\%)$ & 16.2 & 12.8 & 16.8 & 33.3 & 10.0 & 9.1 & 22.4 & 24.4 & 6.0 & 4.5 & 11.8 & 20.7 & 13.0 \\
\hline HS11 & AVG & 0.132 & 0.170 & 0.659 & 3.136 & 0.861 & 0.653 & 2.420 & 2.080 & 24.520 & 37.360 & 1.662 & 3.150 & 0.270 \\
\hline & STD & 0.009 & 0.010 & 0.169 & 0.234 & 0.095 & 0.080 & 0.359 & 0.717 & 1.436 & 1.159 & 0.228 & 0.891 & 0.013 \\
\hline & $\operatorname{cov}(\%)$ & 7.2 & 5.9 & 25.6 & 7.5 & 11.0 & 12.3 & 14.8 & 34.5 & 5.9 & 3.1 & 13.7 & 28.3 & 4.8 \\
\hline HS11 & AVG & 0.174 & 0.214 & 0.682 & 3.032 & 0.895 & 0.666 & 2.290 & 2.172 & 23.860 & 35.700 & 1.648 & 3.462 & 0.326 \\
\hline & STD & 0.017 & 0.019 & 0.173 & 0.297 & 0.055 & 0.045 & 0.285 & 0.529 & 2.110 & 1.828 & 0.139 & 0.420 & 0.023 \\
\hline & $\operatorname{cov}(\%)$ & 9.7 & 8.7 & 25.3 & 9.8 & 6.2 & 6.8 & 12.4 & 24.3 & 8.8 & 5.1 & 8.4 & 12.1 & 7.1 \\
\hline HS12 & AVG & 0.145 & 0.185 & 0.582 & 2.928 & 0.859 & 0.643 & 2.644 & 2.066 & 24.840 & 36.120 & 1.720 & 3.160 & 0.298 \\
\hline & STD & 0.006 & 0.008 & 0.105 & 0.233 & 0.076 & 0.093 & 0.515 & 0.710 & 1.936 & 2.306 & 0.169 & 0.837 & 0.016 \\
\hline & $\operatorname{COV}(\%)$ & 4.5 & 4.1 & 18.1 & 8.0 & 8.9 & 14.4 & 19.5 & 34.4 & 7.8 & 6.4 & 9.8 & 26.5 & 5.3 \\
\hline HS12 & AVG & 0.164 & 0.205 & 0.728 & 3.044 & 0.847 & 0.644 & 2.292 & 1.684 & 23.380 & 35.580 & 1.538 & 2.932 & 0.334 \\
\hline & STD & 0.017 & 0.018 & 0.143 & 0.351 & 0.085 & 0.079 & 0.221 & 0.349 & 2.956 & 2.376 & 0.136 & 0.403 & 0.027 \\
\hline & $\operatorname{COV}(\%)$ & 10.3 & 9.0 & 19.6 & 11.5 & 10.0 & 12.3 & 9.6 & 20.7 & 12.6 & 6.7 & 8.9 & 13.8 & 8.0 \\
\hline HS13 & AVG & 0.139 & 0.181 & 0.577 & 2.860 & 0.834 & 0.657 & 2.502 & 1.841 & 24.640 & 36.600 & 1.646 & 3.072 & 0.292 \\
\hline & STD & 0.010 & 0.012 & 0.073 & 0.231 & 0.042 & 0.051 & 0.383 & 0.449 & 1.613 & 1.723 & 0.163 & 0.697 & 0.025 \\
\hline & $\operatorname{COV}(\%)$ & 6.9 & 6.4 & 12.6 & 8.1 & 5.0 & 7.7 & 15.3 & 24.4 & 6.5 & 4.7 & 9.9 & 22.7 & 8.5 \\
\hline HS13 & AVG & 0.150 & 0.192 & 0.593 & 3.002 & 0.883 & 0.646 & 2.814 & 2.334 & 23.840 & 36.360 & 1.666 & 3.296 & 0.315 \\
\hline & STD & 0.033 & 0.037 & 0.113 & 0.364 & 0.082 & 0.087 & 0.339 & 0.807 & 1.460 & 1.916 & 0.146 & 0.480 & 0.065 \\
\hline & $\operatorname{COV}(\%)$ & 21.9 & 19.5 & 19.0 & 12.1 & 9.3 & 13.4 & 12.1 & 34.6 & 6.1 & 5.3 & 8.8 & 14.6 & 20.7 \\
\hline LG11 & AVG & 0.142 & 0.188 & 0.737 & 3.583 & 0.691 & 0.499 & 1.491 & 0.841 & 24.880 & 37.360 & 2.464 & 1.641 & 0.287 \\
\hline & STD & 0.023 & 0.026 & 0.187 & 0.659 & 0.066 & 0.051 & 0.159 & 0.142 & 2.542 & 2.225 & 0.260 & 0.191 & 0.040 \\
\hline & $\operatorname{cov}(\%)$ & 16.1 & 13.7 & 25.4 & 18.4 & 9.6 & 10.2 & 10.7 & 16.8 & 10.2 & 6.0 & 10.6 & 11.6 & 14.0 \\
\hline LG11 & AVG & 0.147 & 0.192 & 0.632 & 3.219 & 0.698 & 0.505 & 1.495 & 0.849 & 27.060 & 39.230 & 2.390 & 1.660 & 0.296 \\
\hline & STD & 0.024 & 0.025 & 0.144 & 0.510 & 0.094 & 0.069 & 0.364 & 0.259 & 2.546 & 2.520 & 0.353 & 0.394 & 0.042 \\
\hline & $\operatorname{COV}(\%)$ & 16.6 & 12.8 & 22.9 & 15.8 & 13.4 & 13.6 & 24.4 & 30.5 & 9.4 & 6.4 & 14.8 & 23.7 & 14.1 \\
\hline LP11 & AVG & 0.183 & 0.240 & 0.852 & 3.665 & 0.660 & 0.503 & 1.268 & 0.761 & 35.280 & 47.810 & 2.775 & 1.451 & 0.340 \\
\hline & STD & 0.018 & 0.018 & 0.116 & 0.540 & 0.065 & 0.050 & 0.273 & 0.163 & 2.518 & 2.417 & 0.461 & 0.287 & 0.024 \\
\hline & $\operatorname{COV}(\%)$ & 10.1 & 7.5 & 13.6 & 14.7 & 9.9 & 9.9 & 21.5 & 21.4 & 7.1 & 5.1 & 16.6 & 19.8 & 7.2 \\
\hline LP11 & AVG & 0.180 & 0.238 & 0.940 & 4.143 & 0.633 & 0.469 & 1.312 & 0.731 & 34.470 & 46.990 & 2.893 & 1.455 & 0.354 \\
\hline & STD & 0.024 & 0.025 & 0.112 & 0.453 & 0.058 & 0.045 & 0.283 & 0.153 & 2.275 & 2.524 & 0.417 & 0.277 & 0.046 \\
\hline & $\operatorname{COV}(\%)$ & 13.5 & 10.4 & 11.9 & 10.9 & 9.1 & 9.6 & 21.6 & 20.9 & 6.6 & 5.4 & 14.4 & 19.0 & 13.0 \\
\hline
\end{tabular}


TABLE 3-Texture classification for geomembranes.

\begin{tabular}{lcccc}
\hline Texture Level & $P_{a}, \mathrm{~mm}$ & $P S_{m}, \mathrm{~mm}$ & $P_{\Delta a}$, Degree & $P_{\lambda_{q}}, \mathrm{~mm}$ \\
\hline Low & $<0.14$ & $>1.9$ & $<26$ & $>2.7$ \\
Moderate & $0.14-0.18$ & $1.4-1.9$ & $26-32$ & $1.7-2.7$ \\
High & $>0.18$ & $<1.4$ & $>32$ & $<1.7$ \\
\hline
\end{tabular}

TABLE 4-Isotropy analysis.

\begin{tabular}{lcccc}
\hline Sample & $P_{a}(\mathrm{MD} / \mathrm{CM})^{a}$ & $P_{\triangle a}(\mathrm{MD} / \mathrm{CM})$ & $P_{\lambda_{q}}(\mathrm{MD} / \mathrm{CM})$ & $P P_{c}(\mathrm{MD} / \mathrm{CM})$ \\
\hline HG11 & 1.062 & 1.039 & 0.978 & 1.162 \\
HG12 & 1.001 & 0.906 & 0.969 & 0.970 \\
HM11 & 0.867 & 1.101 & 1.027 & 1.079 \\
HM12 & 0.864 & 1.012 & 0.893 & 0.997 \\
HP11 & 0.785 & 1.004 & 1.175 & 1.026 \\
HP12 & 1.015 & 1.042 & 1.091 & 1.048 \\
HS11 & 0.763 & 1.028 & 0.910 & 1.008 \\
HS12 & 0.880 & 1.062 & 1.078 & 1.118 \\
HS13 & 0.927 & 1.034 & 0.932 & 0.988 \\
LG11 & 0.967 & 0.919 & 0.988 & 1.031 \\
LP11 & 1.017 & 1.023 & 0.998 & 0.959 \\
\hline
\end{tabular}

${ }^{a}$ Machine Direction/Cross Machine Direction.

TABLE 5-Directionality analysis.

\begin{tabular}{|c|c|c|c|c|}
\hline Sample & $\begin{array}{c}\text { Positive Slopes, } \\
\%\end{array}$ & $\begin{array}{c}\text { Negative Slopes, } \\
\%\end{array}$ & $\begin{array}{l}\text { Directionality } \\
\text { Ratio }\end{array}$ & $\begin{array}{c}\text { Variation } \\
\%\end{array}$ \\
\hline HG11 (MD) & 50.12 & 49.88 & 1.01 & 0.48 \\
\hline HG11 (CM) & 57.45 & 42.55 & 1.35 & 25.94 \\
\hline HG12 (MD) & 47.82 & 52.18 & 0.92 & -8.36 \\
\hline HG12 (CM) & 61.83 & 38.17 & 1.62 & 38.27 \\
\hline HM11 (MD) & 51.89 & 48.11 & 1.08 & 7.28 \\
\hline HM1 1 (CM) & 59.81 & 40.19 & 1.49 & 32.80 \\
\hline HM12 (MD) & 46.55 & 53.45 & 0.87 & -12.91 \\
\hline HM12 (CM) & 61.86 & 38.14 & 1.62 & 38.34 \\
\hline HP11 (MD) & 55.55 & 44.45 & 1.25 & 19.98 \\
\hline HP11 (CM) & 46.35 & 53.65 & 0.86 & -13.61 \\
\hline HP12 (MD) & 57.84 & 42.16 & 1.37 & 27.11 \\
\hline HP12 (CM) & 42.56 & 57.44 & 0.74 & -25.91 \\
\hline HS11 (MD) & 48.70 & 51.30 & 0.95 & -5.07 \\
\hline HS11 (CM) & 60.60 & 39.40 & 1.54 & 34.98 \\
\hline HS12 (MD) & 57.06 & 42.94 & 1.33 & 24.75 \\
\hline $\mathrm{HS} 12$ (CM) & 62.10 & 37.90 & 1.64 & 38.97 \\
\hline HS13 (MD) & 45.34 & 54.66 & 0.83 & -17.05 \\
\hline HS13 (CM) & 55.70 & 44.30 & 1.26 & 20.47 \\
\hline LG11 (MD) & 44.83 & 55.17 & 0.81 & -18.74 \\
\hline LG11 (CM) & 55.88 & 44.12 & 1.27 & 21.05 \\
\hline LP11 (MD) & 58.06 & 41.94 & 1.38 & 27.76 \\
\hline LP11 (CM) & 43.88 & 56.12 & 0.78 & -21.81 \\
\hline
\end{tabular}

TABLE 6-Thicknesses determined using image analysis.

\begin{tabular}{|c|c|c|c|c|c|c|c|c|c|c|c|}
\hline Statistic & HG11 & HG12 & HM11 & HM12 & HP11 & HP12 & HS11 & HS12 & HS13 & LG11 & LP11 \\
\hline AVG (mm) & 1.220 & 1.727 & 1.225 & 1.747 & 1.795 & 2.267 & 1.201 & 1.700 & 2.702 & 1.200 & 1.249 \\
\hline STD (mm) & 0.029 & 0.026 & 0.035 & 0.037 & 0.032 & 0.025 & 0.052 & 0.047 & 0.057 & 0.037 & 0.038 \\
\hline $\operatorname{cov}(\%)$ & 2.4 & 1.5 & 2.9 & 2.1 & 1.8 & 1.1 & 4.3 & 2.8 & 2.1 & 3.1 & 3.0 \\
\hline Skewness & 1.141 & 1.070 & 0.881 & 1.217 & 1.296 & 1.232 & 1.332 & 1.203 & 1.234 & 1.121 & 1.309 \\
\hline Kurtosis & 5.179 & 4.795 & 4.550 & 5.137 & 5.839 & 6.184 & 6.049 & 5.537 & 5.698 & 5.100 & 5.702 \\
\hline Range (mm) & $0.9-2.0$ & $1.4-2.6$ & $0.9-2.2$ & $1.5-2.6$ & $1.4-2.5$ & $1.9-3.1$ & $0.9-2.1$ & $1.4-2.5$ & $2.4-3.6$ & $0.9-2.0$ & $0.9-2.0$ \\
\hline Percent $<\mathrm{NT}^{a}$ & 4.85 & 3.11 & 1.28 & 1.65 & 7.13 & 7.53 & 7.14 & 5.86 & 5.49 & 3.02 & 4.04 \\
\hline $80 \%$ Range $(\mathrm{mm})$ & $1.0-1.4$ & $1.5-1.9$ & $1.0-1.5$ & $1.5-1.9$ & $1.5-2.0$ & $2.0-2.5$ & $1.0-1.5$ & $1.5-2.0$ & $2.5-3.1$ & $1.0-1.4$ & $1.0-1.4$ \\
\hline
\end{tabular}

${ }^{a}$ Nominal Thickness. 

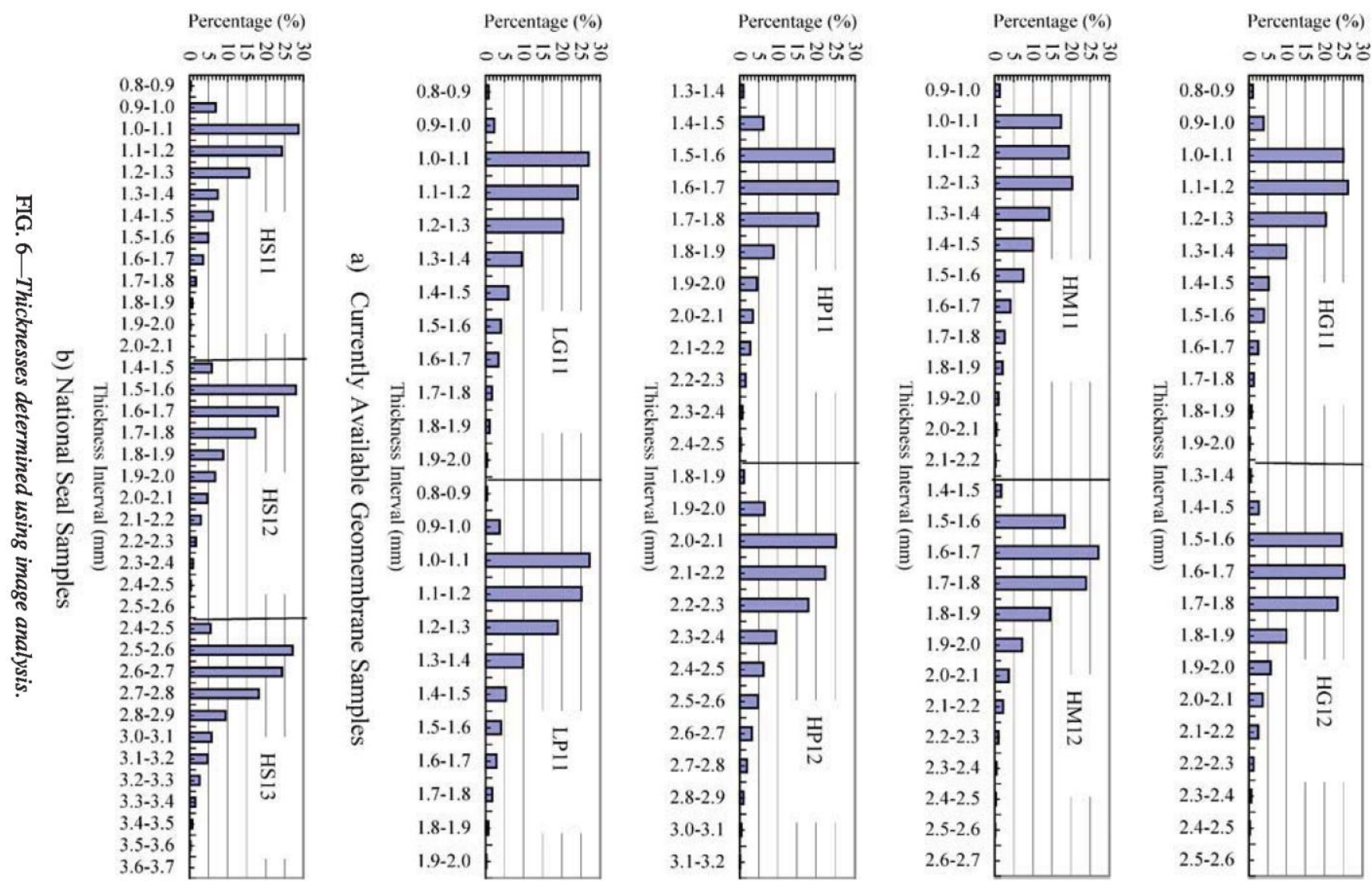
TABLE 7-Core thickness measurements.

\begin{tabular}{|c|c|c|c|c|c|c|c|c|c|c|c|}
\hline Statistic & HG11 & HG12 & HM11 & HM12 & HP11 & HP12 & HS11 & HS12 & HS13 & LG11 & LP11 \\
\hline $\mathrm{AVG}_{\text {mech }}(\mathrm{mm})$ & 1.066 & 1.579 & 1.178 & 1.656 & 1.501 & 1.974 & 1.035 & 1.534 & 2.526 & 1.116 & 1.099 \\
\hline $\mathrm{STD}_{\text {mech }}(\mathrm{mm})$ & 0.082 & 0.060 & 0.061 & 0.050 & 0.048 & 0.047 & 0.056 & 0.055 & 0.056 & 0.056 & 0.064 \\
\hline $\operatorname{COV}_{\text {mech }}(\%)$ & 7.7 & 3.8 & 5.2 & 3.0 & 3.2 & 2.4 & 5.4 & 3.6 & 2.2 & 5.1 & 5.8 \\
\hline $\mathrm{AVG}_{\text {image }}(\mathrm{mm})$ & 0.865 & 1.396 & 0.943 & 1.459 & 1.385 & 1.859 & 0.900 & 1.410 & 2.411 & 0.874 & 0.889 \\
\hline $\mathrm{STD}_{\text {image }}(\mathrm{mm})$ & 0.030 & 0.038 & 0.021 & 0.016 & 0.035 & 0.032 & 0.018 & 0.007 & 0.006 & 0.034 & 0.035 \\
\hline $\mathrm{COV}_{\text {image }}(\%)$ & 3.5 & 2.7 & 2.2 & 1.1 & 2.5 & 1.7 & 2.0 & 0.5 & 0.3 & 3.8 & 4.0 \\
\hline Variation (\%) & 18.83 & 11.58 & 19.99 & 11.94 & 7.71 & 5.83 & 13.01 & 8.10 & 4.56 & 21.69 & 19.15 \\
\hline
\end{tabular}

TABLE 8-Asperity height measurements.

\begin{tabular}{|c|c|c|c|c|c|c|c|c|c|c|c|}
\hline Statistic & HG11 & HG12 & HM11 & HM12 & HP11 & HP12 & HS11 & HS12 & HS13 & LG11 & LP11 \\
\hline $\mathrm{AVG}_{\mathrm{Mech}}(\mathrm{mm})$ & 0.377 & 0.454 & 0.599 & 0.599 & 0.597 & 0.695 & 0.630 & 0.704 & 0.646 & 0.534 & 0.480 \\
\hline $\mathrm{STD}_{\text {Mech }}(\mathrm{mm})$ & 0.074 & 0.061 & 0.041 & 0.032 & 0.041 & 0.060 & 0.098 & 0.074 & 0.087 & 0.052 & 0.030 \\
\hline $\mathrm{COV}_{\text {Mech }}(\%)$ & 19.7 & 13.5 & 6.9 & 5.4 & 6.9 & 8.7 & 15.5 & 10.6 & 13.4 & 9.8 & 6.2 \\
\hline $\mathrm{AVG}_{\text {Image }}(\mathrm{mm})$ & 0.627 & 0.777 & 0.790 & 0.788 & 0.858 & 0.901 & 0.878 & 0.853 & 0.859 & 0.695 & 0.646 \\
\hline $\mathrm{STD}_{\text {Image }}(\mathrm{mm})$ & 0.075 & 0.097 & 0.086 & 0.117 & 0.055 & 0.101 & 0.084 & 0.085 & 0.073 & 0.083 & 0.065 \\
\hline $\mathrm{COV}_{\text {Image }}(\%)$ & 12.0 & 12.5 & 10.9 & 14.8 & 6.4 & 11.3 & 9.5 & 10.0 & 8.5 & 12.0 & 10.0 \\
\hline Variation (\%) & 39.80 & 41.51 & 24.18 & 23.98 & 30.37 & 22.84 & 28.27 & 17.44 & 24.75 & 23.10 & 25.70 \\
\hline
\end{tabular}

